\title{
Uso de substâncias psicoativas entre estudantes de Goianá, MG
}

\author{
Andréia Fernandes Teixeira \\ Secretaria Municipal de Saúde - Goianá \\ Poliana Patrício Aliane \\ Universidade de São Paulo - Ribeirão Preto \\ Luiz Claudio Ribeiro \\ Telmo Mota Ronzani \\ Universidade Federal de Juiz de Fora
}

\begin{abstract}
Resumo
O objetivo foi avaliar o consumo de substâncias psicoativas entre estudantes, do ensino fundamental e médio, da rede pública de ensino do município de Goianá, Minas Gerais. Foi realizado um estudo descritivo, transversal, utilizando um instrumento desenvolvido pela Organização Mundial da Saúde. Participaram 415 estudantes, 57,1\% do Ensino Fundamental e 42,9\% do Ensino Médio, sendo 49,4\% do sexo feminino e 47,5\% do masculino, predominando a faixa etária de 13 a 15 anos e a classe social C. O álcool foi a substância mais utilizada entre os jovens, tendo uso inicial precoce, assim como nos levantamentos nacionais. Sobre o uso na vida, as substâncias mais relatadas foram: solventes, anfetamínicos, ansiolíticos, maconha e alucinógenos. As mulheres apresentaram maior padrão de uso de substâncias psicoativas ilícitas na vida, no ano, no mês, uso freqüente e uso pesado. Em comparação com a capital mineira, foi observado um menor uso de maconha e cocaína entre os estudantes de Goianá. A pesquisa aponta a necessidade de formulação de políticas públicas, considerando particularidades locais e a importância de novos estudos que ampliem a discussão sobre o uso de substâncias psicoativas.
\end{abstract}

Palavras-chave: epidemiologia; estudantes; psicotrópicos

\begin{abstract}
Use of psychoactive substances among students from Goianá, MG, Brazil. The aim was to evaluate the prevalence of the consumption of psychoactive substances among students from the public school in Goianá, Minas Gerais. A descriptive, cross sectional study was performed using a structured questionnaire developed by the World Health Organization. The participants were 415 students, $57.1 \%$ were elementary school students and $42.9 \%$ were high school students. $49.4 \%$ were female and $47.5 \%$ were male. The majority of the students were 13-15 years old and from the $\mathrm{C}$ social class. Alcohol was the substance most frequently used among the young people (64.6\%), as the prevalence founded in the national sample. The main used substances were: solvents (11.1\%), amphetamines (2.9\%), anxyolitics (2.9\%), cannabis (2.7\%) and hallucinogens (1.4\%). Women presented a higher pattern of use for the illicit psychoactive substances. The Goianá students presented a lower consumption of marijuana and cocaine in comparison with students from Belo Horizonte, the state capital. The epidemiologic research points to the necessity of formulating public policies, considering local particularities.
\end{abstract}

Keywords: epidemiology; students; psychotropic drugs

$\mathrm{O}$ uso de substâncias psicoativas por adolescentes decorre de múltiplas variáveis e deve ser visto sob um aspecto biopsicossocial (Babor et al., 2003; DeMicheli \& Formigoni, 2003; Galduróz, Noto \& Carlini, 1997; Papalia \& Olds, 2000). Conhecer a realidade do uso de psicoativos entre adolescentes por meio de seu auto-relato, reunindo dados epidemiológicos acerca do assunto, é uma importante ferramenta para a formulação e avaliação de políticas públicas adequadas destinadas à prevenção, redução de danos e tratamento de dependentes dessas substâncias (Carlini-Cotrim \& Barbosa,
1993; Noto, 2004). É importante que tais levantamentos sejam realizados com regularidade e numa perspectiva de avaliação regional para se acompanhar a evolução do comportamento frente ao uso de substâncias psicoativas em diferentes momentos e realidades sociais (Carlini-Cotrim \& Barbosa, 1993; Galduróz, Noto, Fonseca \& Carlini, 2005; Noto, 2004; Galduróz et al., 1997).

As conseqüências do uso de substâncias psicoativas variam de acordo com o tipo, a dosagem e interação da substância utilizada, e apresentam uma evolução mais rápida entre os 
adolescentes se comparados com indivíduos adultos (Júnior, 1998). O abuso de substâncias psicoativas por jovens brasileiros inclui a mortalidade, entre 15 a 25 anos no Brasil, entre as dez mais altas do mundo (OMS, 2001). Além disso, pode desencadear episódios de violência domiciliar e criminalidade (WHO, 2002), e estar relacionado com internações, acidentes, brigas, evasão escolar, e outros problemas que acarretarão conseqüências secundárias, incluindo o desenvolvimento de doenças (Babor et al., 2003).

No Brasil, pesquisadores empenham esforços em estudo de abrangência regional (Baus, Kupek \& Pires, 2002; Tavares, Béria \& Lima, 2001) e nacional sobre diversos aspectos das drogas (Galduróz et al., 1997; Galduróz et al., 2005). Os estudos com adolescentes, anteriores a 1986 não permitem comparações de resultados por empregarem diferentes metodologias. A partir deste ano, porém, a elaboração de um questionário pela "WHO Research and Reporting Project on the Epidemiology of Drug Dependence”, proposto pela Organização Mundial de Saúde (OMS) e adaptado para o uso no Brasil por Carlini-Cotrim et al. (1987) possibilitou a padronização destes estudos (Galduróz et al., 1997; Tavares et al., 2001).

Dados epidemiológicos nacionais vêm sendo obtidos por meio dos estudos realizados pelo Centro Brasileiro de Informações sobre Drogas Psicotrópicas - CEBRID - do Departamento de Psicobiologia da Universidade Federal de São Paulo. Desde 1987, o CEBRID realiza o levantamento sobre o uso de drogas psicotrópicas entre estudantes de escolas públicas em capitais brasileiras (Galduróz et al., 1997; Galduróz et al., 2005). Em 2004 foi realizado o V Levantamento, abrangendo as 27 capitais.

A importância de estudos epidemiológicos sobre o uso de drogas, para a estruturação de políticas públicas adequadas e racionais, é apontada em estudos nacionais e internacionais (Carlini-Cotrim \& Barbosa, 1993; Galduróz et al., 1997). Segundo Galduróz et al. (1997), traçar o diagnóstico e perfil do uso de drogas contribui ainda para evitar as distorções observadas neste campo e, portanto, os alardes sociais a partir de casos isolados como pode ser observado nas veiculações de informações sobre drogas pela mídia. Segundo Noto et al. (2003) ainda não há distribuição equilibrada das notícias veiculadas na mídia com os indicadores epidemiológicos, “essa desproporção reflete-se diretamente na percepção da população, distorcendo as crenças relativas ao uso de psicotrópicos no País” (p.76), e a formulação de políticas públicas.

Nesse sentido, é importante que além dos estudos de abrangência nacional, também sejam realizados estudos regionais que utilizem a mesma metodologia trazendo à tona peculiaridades que possam estar influenciando o comportamento frente ao uso de drogas. Em levantamento realizado em 2002, em uma escola pública de Florianópolis, constatou-se que a prevalência do uso na vida, de maconha, solventes, anfetamínicos e álcool, foi elevada se comparada aos dados obtidos pelo CEBRID, 1997, em outras capitais da região sul e à média brasileira (Baus et al., 2002). A capital é a segunda destinação de turistas brasileiros e tem alta incidência de AIDS, cuja principal forma de transmissão é o uso de drogas injetáveis (Baus et al.,
2002). Embora não se possam generalizar os dados para os adolescentes do município, devido à restrição da amostra, ressalta a importância de realização de estudos regionais.

Mediante o exposto, o objetivo desta pesquisa foi realizar um censo do uso de substâncias psicoativas entre adolescentes, estudantes, matriculados a partir da $5^{\text {a }}$ série do ensino fundamental e no ensino médio, no município de Goianá, Minas Gerais. O município está localizado na Zona da Mata de Minas Gerais, com população estimada de 3.643 habitantes e que tem como principal atividade econômica a prestação de serviços, com um produto interno bruto per capita é de $\mathrm{R} \$ 4.775,00$ e com índice de desenvolvimento humano de 0,74 (médio). A cidade conta com 1 Unidade Básica de Saúde, com referenciamento de serviços especializados para o município de Juiz de Fora.

\section{Método}

\section{Participantes}

A população-alvo, desta pesquisa, foram 415 estudantes do ensino fundamental, a partir da $5^{\mathrm{a}}$ série, e do ensino médio da rede pública de ensino do município. Em Goianá, apenas uma escola oferece ensino fundamental e médio e portando, optou-se pela realização de um estudo censitário.

\section{Instrumentos}

O instrumento utilizado foi um questionário fechado, de autopreenchimento, sem identificação pessoal do aluno, traduzido e adaptado por Carlini-Cotrim et al. (1987), desenvolvido pela WHO Research and Reporting Project on the Epidemiology of Drug Dependence, apresentado no documento "A Metodology for Student Drug-Use Survey” e proposto pela OMS (CarliniCotrim \& Barbosa, 1993; Galduróz et al., 1997). O questionário é composto de três partes: (1) dados sócio-demográficos; (2) comportamento de uso de drogas e; (3) questões opcionais que objetivam coletar dados sobre a vida do estudante e sua família, fornecendo subsídios para realização de estudos posteriores sobre as variáveis do uso de drogas no Brasil (Carlini-Cotrim \& Barbosa, 1993). Os dados sócio-demográficos avaliados no instrumento baseiam-se na escala sócio-econômica proposta pela ABIPEME - Associação Brasileira de Institutos de Pesquisa de Mercado.

Este instrumento vem sendo utilizado nos levantamentos nacionais realizados pelo CEBRID no Brasil, sendo utilizado na presente pesquisa a mesma versão do V Levantamento Nacional realizado em 2004.

\section{Procedimentos}

Para fins de comparação, esta pesquisa replicou a metodologia utilizada nos levantamentos nacionais realizados pelo Centro Brasileiro de Informações de Drogas Psicotrópicas - CEBRID (Galduróz et al., 1997). Inicialmente, o projeto foi apresentado à Secretaria de Estado da Educação - 18 Superintendência Regional de Ensino, localizada no município de Juiz de Fora, e à Prefeitura Municipal de Goianá, sendo solicitada autorização para a realização da pesquisa na rede pública de ensino do município. A direção da escola do município 
de Goianá foi contatada para apresentação do projeto e convidada a participar, voluntariamente, da pesquisa. Foi realizado agendamento da aplicação dos questionários e assinatura do termo de consentimento, em duas vias, relativo à participação da escola e de seus alunos no referido projeto. O projeto contou com a aprovação pelo Comitê de Ética em Pesquisa da Universidade Federal de São Paulo, para execução em todo país, estando aprovado sob o registro CEP $\mathrm{N}^{\underline{0}}$ 0718/03.

A presente pesquisa contou com a participação de seis aplicadores, previamente treinados, de forma que todo o procedimento fosse realizado em única visita à escola, atendendo a metodologia proposta pelo CEBRID (Carlini-Cotrim \& Barbosa, 1993). A realização da aplicação dos questionários ocorreu em março de 2005, em sala de aula, coletivamente, sem a presença de professores, após breve explicação dos objetivos do trabalho aos alunos. O preenchimento do questionário pelo aluno foi facultativo tendo sido reservada a liberdade de devolvêlo em branco. A fim de garantir o anonimato, a devolução dos questionários consistiu no depósito dos mesmos, pelo aluno, em uma urna lacrada.

\section{Análise estatística}

Antes de procedermos às análises estatísticas dos dados, foi realizada a microcrítica, por meio de inspeção dos questionários individualmente, com objetivo de detectar erros por negligência ou interpretação, o que possibilitou a realização da crítica qualitativa por meio de avaliação da coerência interna das respostas. Todos os questionários que apresentaram questões incoerentes foram listados para verificação por três pesquisadores, em separado, cuja avaliação decidiria pela anulação total ou parcial das mesmas ou pela alteração da resposta (caso estivesse clara a intenção do respondente). Nestes dois últimos casos, deveria haver consenso entre os pesquisadores. Essa medida seguiu as orientações propostas pelo Manual de Orientações Gerais (Carlini-Cotrim \& Barbosa, 1993).

Os dados foram digitados e analisados no software estatístico SPSS, versão 15.0, sendo realizadas análises descritivas das variáveis estudadas. Por se tratar de um censo, as diferenças encontradas são reais, pois se referem a toda a população pesquisada.

A variável uso de substâncias psicoativas foi definida segundo critérios da OMS, conforme transcrito:

Uso na vida: quando a pessoa fez uso de qualquer droga pelo menos uma vez na vida;

Uso no ano: quando a pessoa utilizou droga pelo menos uma vez nos doze meses que antecederam à pesquisa;

Uso no mês: quando a pessoa utilizou droga pelo menos uma vez nos trinta dias que antecederam à pesquisa;

Uso freqüente: quando a pessoa utilizou droga seis vezes ou mais nos trinta dias em que antecederam à pesquisa;

Uso pesado: quando a pessoa utilizou droga vinte vezes ou mais nos trinta dias em que antecederam à pesquisa.

Para fins de entendimento, faz-se necessário a definição de não-usuário, segundo a classificação da OMS. Não-usuário: nunca utilizou drogas.

Neste estudo, foram consideradas substâncias psicoativas de uso ilícito: maconha, cocaína, crack, solventes, opiáceos, alucinógenos, orexígenos, anticolinérgicos, esteróides e anabolizantes. Com relação aos medicamentos (substância psicoativas lícitas), tais como anfetamínicos, ansiolíticos, xaropes, barbitúricos, foi pesquisado o uso ilícito dos mesmos, ou seja, sem prescrição médica.

\section{Resultados}

Dos 415 estudantes pesquisados $49,4 \%$ pertenciam ao sexo feminino, $47,5 \%$ ao sexo masculino e 3,1\% não informaram o sexo. Entre os participantes da pesquisa, a maior concentração de estudantes correspondeu à faixa etária de 13 a 15 anos (Tabela 1).

No momento da pesquisa $57,1 \%$ dos estudantes estavam matriculados no ensino fundamental e 42,9\% no ensino médio. Com relação à defasagem escolar, mensurada pela diferença entre idade e série, observou-se que $72,8 \%$ não apresentaram defasagem escolar (Tabela 1).

Tabela 1

Características sócio-demográficas de 415 estudantes do ensino fundamental e médio da rede pública de Goianá.

\begin{tabular}{llrr}
\hline \multicolumn{1}{c}{ Características } & & $n$ & $\%$ \\
\hline \multirow{2}{*}{ Sexo } & Masculino & 197 & 47,5 \\
& Feminino & 205 & 49,4 \\
& Não informado & 13 & 3,1 \\
\cline { 2 - 4 } Total & 415 & 100,0 \\
\hline \multirow{2}{*}{ Faixa etária (anos) } & 10 a 12 & 102 & 24,6 \\
& 13 a 15 & 187 & 45,1 \\
& 16 a 18 & 100 & 24,1 \\
& $>18$ & 14 & 3,4 \\
& Não informado & 12 & 2,9 \\
\cline { 2 - 4 } Notal & 415 & 100,0 \\
\hline \multirow{2}{*}{ Dível escolar } & Fundamental & 237 & 57,1 \\
& Médio & 178 & 42,9 \\
\cline { 2 - 4 } Série/idade (anos) & Total & 415 & 100,0 \\
\cline { 2 - 4 } & Não tem & 302 & 72,8 \\
& 1 a & 76 & 18,3 \\
& Não Informado & 25 & 6,0 \\
\cline { 2 - 4 } Nível sócio- & Total & 12 & 2,9 \\
\hline \multirow{2}{*}{ econômico } & A & 415 & 100,0 \\
& $\mathrm{~B}$ & 34 & 8,2 \\
& $\mathrm{C}$ & 63 & 15,2 \\
& $\mathrm{D}$ & 164 & 39,5 \\
& $\mathrm{E}$ & 69 & 16,6 \\
& Não Informado & 32 & 0,7 \\
\cline { 2 - 4 } & Total & 415 & 100,8 \\
\hline
\end{tabular}

Onível sócio-econômico foi caracterizado pela concentração de 39,5\% dos estudantes na classe C (Tabela 1).

O uso na vida de substâncias psicoativas ilícitas foi relatado por $15,2 \%$ dos estudantes. $\mathrm{O}$ uso no ano foi de $9,4 \%$ e o uso no mês foi de $5,1 \%$. O uso freqüente e o uso pesado foram relatados por 2,4\% e 2,2\% dos estudantes, respectivamente (Tabela 2). Dos 415 estudantes que participaram deste levantamento, 81,9\% não relataram uso de substâncias psicoativas.

As cinco substâncias ilícitas mais utilizadas na vida pelos estudantes foram: solventes $(11,1 \%)$, anfetamínicos $(2,9 \%)$, ansiolíticos (2,9\%), maconha (2,7\%) e alucinógenos (1,4\%). Das substâncias psicoativas lícitas, o álcool foi à substância de maior relato de uso na vida (64,6\%), seguido do tabaco (20,2\%), como 
pode ser observado na tabela 2. Os solventes foram apontados como a substância ilícita mais utilizada no ano (7,5\%), no mês (4,1\%), uso freqüente ( $1,2 \%)$ e uso pesado $(1,2 \%)$, se comparadas às demais substâncias (Tabela 2).

Na comparação entre os sexos, constatou-se que o relato do uso de crack, cocaína e barbitúricos foi exclusivo dos estudantes do sexo masculino. O relato do uso de opiáceos, alucinógenos e orexígenos equiparou-se entre os dois sexos. Por sua vez, o uso de maconha e anticolinérgicos foi maior entre os estudantes do sexo masculino e, o uso de anfetamínicos, solventes, ansiolíticos

Tabela 2

Uso de substâncias psicoativas por 415 estudantes do ensino fundamental e médio da rede pública de Goianá

\begin{tabular}{lccccc}
\hline \multirow{1}{*}{ Drogas } & Na vida & No ano & No mês & Freqüente & Uso Pesado \\
\cline { 2 - 6 } & $\%$ & $\%$ & $\%$ & $\%$ & $\%$ \\
\hline Maconha & 2,7 & 1,0 & 0,5 & 0,0 & 0,0 \\
Cocaína & 0,5 & 0,2 & 0,2 & 0,2 & 0,2 \\
Crack & 0,5 & 0,2 & 0,0 & 0,0 & 0,0 \\
Anfetamínicos & 2,9 & 2,2 & 1,0 & 0,7 & 0,5 \\
Solventes & 11,1 & 7,5 & 4,1 & 1,2 & 1,2 \\
Ansiolíticos & 2,9 & 1,4 & 0,7 & 0,4 & 0,2 \\
Anticolinérgicos & 1,2 & 0,5 & 0,2 & 0,2 & 0,2 \\
Barbitúricos & 0,2 & 0,0 & 0,0 & 0,0 & 0,0 \\
Opiáceos & 0,5 & $---{ }^{-}$ & --- & --- & --- \\
Xaropes & 1,0 & --- & --- & --- & --- \\
Alucinógenos & 1,4 & --- & --- & --- & --- \\
Orexígenos & 0,5 & --- & --- & --- & --- \\
\hline Total de usuários ${ }^{b}$ & 15,2 & 9,4 & 5,1 & 2,4 & 2,2 \\
\hline Tabaco & 20,2 & 12,8 & 6,3 & 2,1 & 1,4 \\
Álcool & 64,6 & 49,6 & 29,2 & 12,8 & 8,2 \\
\hline
\end{tabular}

Nota. Dados expressos em porcentagem, levando-se em conta as categorias de usuários e as diferentes drogas individualmente.

${ }^{a} \mathrm{O}$ pontilhado (---) indica dados não observados.

${ }^{\text {b }}$ Exceto usuários de álcool e tabaco. Vide definição de uso na vida, no ano, no mês, freqüente e pesado, em método.

e xaropes foi mais frequente entre os estudantes do sexo feminino. Considerando todas as substâncias psicoativas ilícitas os estudantes do sexo feminino apresentaram uma prevalência de uso na vida de $16,2 \%$ enquanto que para os estudantes do sexo masculino a prevalência de uso na vida foi de 13,2\%. As mulheres também apresentaram maior prevalência de uso na vida de álcool (65,9\%) comparada aos homens (62,2\%) conforme se verifica na Tabela 3.

Dos estudantes que relataram uso de substâncias psicoativas ilícitas, 6,1\% estavam na faixa etária de 10 a 12 anos de idade e à medida que aumenta a idade aumentou a prevalência do uso de substâncias ilícitas e também o uso de álcool. (Tabela 3).

O uso na vida de substâncias psicoativas ilícitas foi relatado por $13,2 \%$ dos estudantes do sexo masculino e $16,3 \%$ dos

Tabela 3

Uso na vida de substâncias psicoativas por 415 estudantes de ensino fundamental e médio da rede pública de Goianá; dados expressos em porcentagem, levando-se em conta sexo, idade e as diferentes drogas individualmente.

\begin{tabular}{|c|c|c|c|c|c|c|c|c|}
\hline \multirow{2}{*}{ Drogas } & \multicolumn{3}{|c|}{ Sexo (\%) } & \multicolumn{5}{|c|}{ Idade, em anos (\%) } \\
\hline & M & $\mathrm{F}$ & $\mathrm{NI}^{\mathrm{a}}$ & $10-12$ & $13-15$ & $16-18$ & $>18$ & $\mathrm{NI}^{\mathrm{a}}$ \\
\hline Maconha & 4,6 & 1,0 & 0,0 & 0,0 & 2,2 & 6,0 & 7,1 & 0,0 \\
\hline Cocaína & 1,0 & 0,0 & 0,0 & 0,0 & 0,0 & 1,0 & 7,1 & 0,0 \\
\hline Crack & 1,0 & 0,0 & 0,0 & 0,0 & 0,0 & 2,0 & 0,0 & 0,0 \\
\hline Anfetamínicos & 2,6 & 2,9 & 7,7 & 0,0 & 2,7 & 5,1 & 7,1 & 8,3 \\
\hline Solventes & 8,7 & 11,7 & 41,7 & 5,9 & 11,2 & 14,0 & 7,1 & 36,4 \\
\hline Ansiolíticos & 1,5 & 4,4 & 0,0 & 1,0 & 2,7 & 5,1 & 7,1 & 0,0 \\
\hline Anticolinérgicos & 1,5 & 1,0 & 0,0 & 0,0 & 0,5 & 4,0 & 0,0 & 0,0 \\
\hline Barbitúricos & 0,5 & 0,0 & 0,0 & 0,0 & 0,0 & 1,0 & 0,0 & 0,0 \\
\hline Opiáceos & 0,5 & 0,5 & 0,0 & 0,0 & 0,5 & 1,0 & 0,0 & 0,0 \\
\hline Xaropes & 0,5 & 1,0 & 7,7 & 0,0 & 1,6 & 1,0 & 0,0 & 0,0 \\
\hline Alucinógenos & 1,5 & 1,5 & 0,0 & 0,0 & 2,1 & 2,0 & 0,0 & 0,0 \\
\hline Orexígenos & 0,5 & 0,5 & 0,0 & 0,0 & 0,5 & 1,0 & 0,0 & 0,0 \\
\hline Total de usuários ${ }^{b}$ & 13,2 & 16,3 & 41,7 & 6,1 & 16,4 & 19,6 & 28,6 & 36,4 \\
\hline Tabaco & 23,0 & 18,0 & 15,4 & 2,9 & 17,7 & 45,0 & 14,3 & 8,3 \\
\hline Álcool & 62,2 & 65,9 & 84,6 & 32,4 & 66,7 & 89,0 & 92,9 & 75,0 \\
\hline
\end{tabular}

a NI significa dados não informados pelos alunos

${ }^{\text {b }}$ Para definição de usuários, vide método. 
estudantes do sexo feminino. As estudantes do sexo feminino apresentaram maior padrão de uso de substâncias psicoativas ilícitas na vida, no ano, no mês, uso freqüente e no uso pesado. Dos estudantes que não informaram o sexo, 41,7\% relataram uso na vida de substâncias psicoativas ilícitas (Tabela 4). Em relação ao uso de substâncias psicoativas ilícitas e a idade, observou-se uma progressão de uso na vida e uso no mês com o aumento da idade. Não houve relato de uso freqüente e uso pesado na faixa etária de 16 a 18 anos (Tabela 4).

Mediante comparação da defasagem escolar constatou-se

Tabela 4

Uso de substâncias psicoativas ilícitas, entre 415 estudantes do Ensino Fundamental e Médio da rede pública de Goianá; dados expressos em porcentagem, levando-se em conta as categorias de usuários, segundo sexo e idade.

\begin{tabular}{|c|c|c|c|c|c|c|c|c|}
\hline \multirow{2}{*}{$\begin{array}{c}\text { Categoria de } \\
\text { Usuários }\end{array}$} & \multicolumn{3}{|c|}{ Sexo $(\%)^{a}$} & \multicolumn{5}{|c|}{ Idade, em anos (\%) ${ }^{a}$} \\
\hline & $\mathrm{M}$ & $\mathrm{F}$ & $\mathrm{NI}^{\mathrm{b}}$ & $10-12$ & $13-15$ & $16-18$ & $>18$ & $\mathrm{NI}^{\mathrm{b}}$ \\
\hline Uso na vida & 13,2 & 16,3 & 41,7 & 6,1 & 16,4 & 19,6 & 28,6 & 36,4 \\
\hline Uso no ano & 5,9 & 11,8 & 33,3 & 3,0 & 11,4 & 9,4 & 21,4 & 27,3 \\
\hline Uso no mês & 3,7 & 5,9 & 18,2 & 1,0 & 6,0 & 6,3 & 7,1 & 20,0 \\
\hline Uso freqüente & 1,5 & 2,9 & 7,7 & 1,0 & 3,7 & 0,0 & 7,1 & 8,3 \\
\hline Uso pesado & 1,0 & 2,9 & 7,7 & 1,0 & 3,2 & 0,0 & 7,1 & 8,3 \\
\hline
\end{tabular}

a A diferença para 100\% em cada categoria representa não-usuários da mesma categoria (por exemplo, 13,2\% de usuários na vida e 86,8\% de não-usuários na vida, para o sexo masculino).

${ }^{\text {b }}$ NI significa dados não informados pelos alunos; por exemplo, entre aqueles que não informaram o sexo, houve $41,7 \%$ deles que fizeram uso na vida de drogas.

que $25 \%$ dos usuários de substâncias psicoativas estavam em defasagem escolar e entre os usuários, 63,5\% não estavam em defasagem escolar. A prevalência de defasagem escolar e de absenteísmo escolar foi maior no grupo que declarou uso na vida de alguma substância psicoativa ilícita (Tabela 5).

Quanto ao nível sócio-econômico constatou-se que 59,5\% dos não-usuários são pertencentes às classes sociais C, D e E. Entre os usuários, $46,1 \%$ pertencem às classes sociais $\mathrm{C}$ e $\mathrm{D}$, e $42,8 \%$ pertencem às classes sociais A e B. Não foram constatados usuários pertencentes à classe social E (Tabela 5).

\section{Discussão}

A importância de levantamentos regionais foi observada a partir de dados encontrados que diferem, em alguns aspectos, dos dados nacionais, da região Sudeste e da capital de Minas Gerais - Belo Horizonte - relatados no V Levantamento realizado pelo CEBRID em 2004 (Galduróz et al., 2005).

Constatou-se que o álcool, seguido pelo tabaco, são as substâncias predominantemente mais utilizadas entre os jovens. Observa-se ainda um início de uso precoce entre os

Tabela 5

O uso na vida de substâncias psicoativas, segundo características sociais de 415 estudantes da rede pública de Goianá.

\begin{tabular}{llrrrr}
\hline \multirow{2}{*}{ Características } & & \multicolumn{2}{c}{ Não-usuário } & \multicolumn{2}{c}{ Usuário } \\
& & $n$ & $\%$ & $n$ & \multicolumn{1}{c}{$\%$} \\
\hline \multirow{2}{*}{ Defasagem } & Não tem & 255 & 75,0 & 40 & 63,5 \\
série/idade (anos) & 1 a 2 & 61 & 17,9 & 12 & 19,0 \\
& $\geq 3$ & 17 & 5,0 & 7 & 11,1 \\
& Não Informada $^{\text {a }}$ & 7 & 2,1 & 4 & 6,4 \\
\cline { 2 - 6 } Total $^{\text {a }}$ & 340 & 100,0 & 63 & 100,0 \\
\hline \multirow{2}{*}{ Dias em que não } & $1-3$ & 76 & 22,4 & 15 & 23,8 \\
compareceu à escola & $4-8$ & 4 & 1,2 & 6 & 9,5 \\
no mês & $\geq 9$ & 5 & 1,5 & 2 & 3,2 \\
& Não faltou & 252 & 74,1 & 37 & 58,7 \\
& Não informado & 3 & 0,9 & 3 & 4,8 \\
\cline { 2 - 6 } Nível sócio-econômico & Total ${ }^{\text {a }}$ & 340 & 100,0 & 63 & 100,0 \\
& A & 19 & 5,6 & 14 & 22,2 \\
& $\mathrm{~B}$ & 48 & 14,1 & 13 & 20,6 \\
& $\mathrm{C}$ & 141 & 41,5 & 18 & 28,6 \\
& $\mathrm{D}$ & 58 & 17,1 & 11 & 17,5 \\
& $\mathrm{E}$ & 3 & 0,9 & 0 & 0,0 \\
& Não Informado & 71 & 20,9 & 7 & 11,1 \\
\cline { 2 - 6 } & Total $^{\text {a }}$ & 340 & 100,0 & 63 & 100,0 \\
\hline
\end{tabular}

${ }^{\text {a }} N=403$, dados faltantes $=12$ (relativos a questões em branco e/ou anuladas, referentes ao uso na vida de substâncias psicoativas). 
estudantes - 10 a 12 anos - , fato que deve ser considerado em ulteriores estudos de intervenção da progressão no uso de drogas, bem como no desenvolvimento de possíveis problemas relacionados (Galduróz et al., 2005; Galduróz et al., 1997; Júnior, 1998). Além disso, como apontado nos levantamentos do CEBRID para as capitais do Brasil quanto para Belo Horizonte (Galduróz et al., 2005), os solventes foram a substância ilícita mais utilizada pelos estudantes em todas as categorias de uso avaliadas. Apesar do uso de solvente ser considerável entre os estudantes e ser prevalente no Brasil, se comparado com outros países (Galduróz et al., 2005), pouca ênfase tem sido dada para tal fato na formulação de campanhas preventivas (Noto et al, 2003; Noto, 2004). Tais dados são importantes, pois podem servir como parâmetros objetivos para formulação de estratégias preventivas e implantação de políticas públicas sobre drogas entre estudantes, além de contestar alguns mitos existentes sobre o uso de algumas substâncias.

Ao comparar os resultados encontrados em relação ao uso na vida de substâncias psicoativas com os dados nacionais de diversas capitais do país e de Belo Horizonte (Galduróz et al., 2005), observou-se que o uso de maconha, cocaína, crack, anfetamínicos, solventes, ansiolíticos, barbitúricos, álcool e tabaco foi maior na capital mineira e em todo o país, se comparado a Goianá. Já os opiáceos, xaropes e alucinógenos tiveram maior prevalência de uso na vida em Goianá. Especula-se que tal diferença poderia ser explicada a partir de dois aspectos principais: a dificuldade de acesso a determinadas drogas e a diferença dos padrões de comportamento entre jovens de pequenos e grandes municípios. Outros estudos, porém, são necessários para se chegar a dados mais conclusivos sobre este aspecto.

Outro dado importante foi a diferença entre gênero, referente ao tipo de substância utilizada e padrão de uso. Na comparação entre os tipos de substâncias psicoativas, os homens consumiram mais maconha, crack, cocaína, anticolinérgicos e barbitúricos e as mulheres consumiram mais anfetamínicos, solventes, ansiolíticos e xaropes. Porém, se considerarmos o total de usuários, há mais mulheres do que homens usuários de substâncias psicoativas ilícitas (16,3\% x 13,2\%, respectivamente). Em Belo Horizonte o total de usuários foi praticamente igual entre homens e mulheres (23,8\% e 23,9\% respectivamente) e no Brasil a prevalência de usuários de substâncias ilícitas foi maior para os estudantes do sexo masculino (25,3\%) se comparado aos estudantes do sexo feminino (21,7\%). Além disso, destacase que as mulheres apresentam um maior padrão de uso de drogas em todas as categorias (uso na vida, no ano, no mês, uso freqüente, uso pesado), diferentemente dos dados nacionais mais recentes (Galduróz et al., 2005). A partir de tal fato, ressalta-se a importância da questão "gênero e uso de drogas" como um fator relevante no estudo e intervenção do uso de drogas e suas conseqüências específicas para o fenômeno observado (Galduróz et al., 2005).

Sobre a questão da defasagem escolar e uso de drogas, no presente estudo, os usuários de substâncias apresentaram maior defasagem e faltaram mais às aulas em comparação com os não-usuários. Tal fato pode ser observado a partir de dados nacionais em que, mesmo em lugares com defasagem escolar baixa, os estudantes que apresentam maior defasagem escolar e faltas são mais propensos a usarem drogas (Galduróz et al., 1997; Galduróz et al., 2005). Porém, é necessária uma análise mais aprofundada sobre tal aspecto, pois a defasagem pode estar relacionada a outras variáveis como, por exemplo, idade, propostas pedagógicas ou outras situações contextuais e não se tem um nexo de causalidade definido.

Podem ser apontadas as seguintes limitações do presente estudo: 1) o número de estudantes que não informaram dados, como sexo e idade, pode limitar algumas comparações realizadas; 2) como qualquer instrumento de auto-relato, apesar de todos os cuidados durante a aplicação dos questionários, alguns estudantes podem ter se sentido pouco à vontade para responder questões sobre uso de drogas, principalmente em se tratando de um município de pequeno porte, ou ainda supervalorizar o consumo de algumas drogas.

\section{Considerações finais}

A pesquisa epidemiológica torna evidente a necessidade de, ao se formular políticas públicas, considerar as particularidades existentes em cada localidade. A realização de levantamentos locais deve orientar a estruturação de ações adequadas atingindo assim os problemas reais e específicos. É importante que se busque a padronização desses levantamentos tomando-se como referência o levantamento nacional para que as diferenças encontradas sejam alvo de análise.

As políticas públicas se tornarão mais efetivas quando forem formuladas a partir de dados empíricos e locais. A adoção de modelos externos ou formulação de políticas com base em dados amplos, por exemplo, dados da capital, ou em mitos, preconceitos e interesses comprometem a efetividade dos procedimentos.

O âmbito escolar favorece a implantação de políticas públicas voltadas aos adolescentes, muito embora seja necessário pensar que muitos estão fora da escola e que políticas devem ser elaboradas não só no sentido de assistir a esses jovens nos aspectos referentes ao uso de drogas, como também de inseri-los no sistema de ensino. É importante, para a efetividade de ações de prevenção ou redução de danos, a adequação à realidade e o planejamento multidisciplinar.

Espera-se que a metodologia e as discussões suscitadas no presente estudo sirvam como um parâmetro para a realização de outras pesquisas que aprofundem alguns aspectos ainda em aberto e ampliem a discussão sobre o uso de substâncias psicoativas entre estudantes.

\section{Agradecimentos}

Ao Centro Brasileiro de Informações sobre Drogas Psicotrópicas - CEBRID - pela disponibilização dos questionários. Aos professores José Carlos Fernandes Galduróz, Ana Regina Noto e Yone Gonçalves de Moura, da Universidade Federal de São Paulo (UNIFESP), pela revisão do artigo. Aos psicólogos Lucas Azevedo Martins, Nelimar Ribeiro de Castro, Paula Almeida, Tatiana Amato e Thiago Pavim, pela realização da coleta de dados, à Secretaria Estadual de Ensino, e em especial à direção das escolas e aos alunos, que colaboraram com sua participação neste estudo. 


\section{Referências}

Babor, T., et al. (2003). Alcohol: no ordinary, commodity. Research and public policy. Oxford: Oxford University Press.

Baus, J., Kupek, E., \& Pires, M. (2002). Prevalência e fatores de risco relacionados ao uso de drogas entre escolares. Revista de Saúde Pública, 36(1), 40-46.

Carlini-Cotrim, B., \& Barbosa, M. T. S. (1993). Pesquisas epidemiológicas sobre o uso de drogas entre estudantes: um manual de orientações gerais. São Paulo: CEBRID.

DeMicheli, D., \& Formigoni, M. L. O. S. (2003). Are reasons for the first use of drugs and family circumstances predictors of future use patterns? Addictive Behaviors, 27, 87-100.

Galduróz, J. C. F., Noto, A. R., \& Carlini, E. A. (1997). IV Levantamento sobre o uso de drogas entre estudantes de $1^{\underline{0}}$ e $2^{\underline{0}}$ graus em 10 capitais brasileiras. São Paulo: CEBRID.

Galduróz, J. C. F., Noto, A. R., Fonseca, A. M., \& Carlini, E. A. (2005). V Levantamento sobre o consumo de drogas psicotrópicas entre estudantes do ensino fundamental e médio da rede pública de ensino nas 27 capitais brasileiras - 2004. São Paulo: CEBRID.
Júnior, A. L. (1998). Enfoque contextual das drogas: aspectos biológicos, culturais e educacionais. In J. G. Aquino (Org.), Drogas na escola: alternativas $e$ práticas ( $2^{\underline{a}}$ ed.; pp. 31-43). São Paulo: Summus.

Noto, A. R. (2004). Os índices de consumo de psicotrópicos entre adolescentes no Brasil. In I. Pinsky, \& M. A. Bessa (Orgs.), Adolescência e drogas (pp. 45-53). São Paulo: Contexto.

Noto, A. R., Baptista, M. C., Faria, S., Nappo, A. S., Galduróz, J. C. F., \& Carlini, E. A. (2003). Drogas e saúde na imprensa brasileira: uma análise de artigos e jornais publicados em revistas. Cadernos de Saúde Pública, 19(1), 69-79.

Organização Mundial de Saúde (OMS) (2001). Relatório sobre a saúde no mundo. Saúde mental: nova concepção, nova esperança. Genebra: Autor.

Papalia, D. E., \& Olds, S. W. (2002). Desenvolvimento humano. Porto Alegre: ArtMed.

Tavares, B. F., Béria, J. U., \& Lima, M. S. (2001). Prevalência do uso de drogas e desempenho escolar entre adolescentes. Revista de Saúde Pública, 35(2), 150-158

World Health Organization (WHO). (2002). World report on violence and health Genebra: Autor.

Andréia Fernandes Teixeira, psicóloga pela Universidade Federal de Juiz de Fora, é diretora do Setor de Psicologia da Prefeitura Municipal de Goianá. Endereço para correspondência: Prefeitura Municipal de Goianá; Secretaria Municipal de Saúde - Setor de Psicologia; Avenida 21 de dezembro, 850 (Centro) ; Goianá, MG; CEP: 36152-000. Telefone: (32) 3274-5192. Fax: (32) 3274-5192. E-mail: olharpsi@hotmail.com Poliana Patrício Aliane, mestre em Medicina (Saúde Mental) pela Universidade de São Paulo, doutoranda na mesma instituição. E-mail: poliana_aliane@yahoo.com.br

Luiz Claudio Ribeiro, doutor em Demografia pela Universidade Federal de Minas Gerais, é professor Adjunto no Departamento de Estatística da Universidade Federal de Juiz de Fora. E-mail: luiz.claudio@ufjf.edu.br Telmo Mota Ronzani, pós-Doutor em Saúde Pública pela Universidade de São Paulo e University of Connecticut Health Center, é professor Adjunto no Departamento de Psicologia da Universidade Federal de Juiz de Fora. E-mail: telmo.ronzani@ufjf.edu.br 